\title{
Questes
}

Revue pluridisciplinaire d'études médiévales

\section{Mélancolie et scatologie : de l'humeur noire aux vents et aux excréments}

\section{Pierre Levron}

\section{(2) OpenEdition \\ 1 Journals}

\section{Édition électronique}

URL : https://journals.openedition.org/questes/2659

DOI : 10.4000/questes.2659

ISSN : 2109-9472

Éditeur

Les Amis de Questes

\section{Édition imprimée}

Date de publication : 15 juin 2011

Pagination : 72-88

ISSN : 2102-7188

\section{Référence électronique}

Pierre Levron, « Mélancolie et scatologie : de l'humeur noire aux vents et aux excréments », Questes [En ligne], 21 | 2011, mis en ligne le 15 janvier 2014, consulté le 07 décembre 2022. URL : http:// journals.openedition.org/questes/2659; DOI : https://doi.org/10.4000/questes.2659

Tous droits réservés 


\section{Mélancolie et scatologie : de l'humeur noire aux vents et aux excréments.}

\section{Pierre LEVRON}

La maladie d'amour va-t-elle de pair avec la scatologie ? La poésie graveleuse peut-elle nous apprendre quelque chose sur la fin'amor ou sur la souffrance amoureuse? Il faut dépasser ce paradoxe apparent né d'une conception éthérée de l'amour courtois. Depuis une trentaine d'années, les spécialistes de la lyrique d'oc et d'oïl ont attiré l'attention sur l'importance des textes à visée scatologique ou qui, du moins, comprennent des passages scatologiques. Les anthologies de René Nelli ${ }^{1}$ et de Pierre Bec $^{2}$ mettent en évidence deux phénomènes : le discours non conventionnel est important pour l'étude de la lyrique d'oc, et la scatologie n'y est pas rare. Les cansos ou les chansons apparaissent, au côté de certains genres plus parodiques (la sotte chanson d'oïl par exemple), comme les types fondamentaux de ces textes burlesques ou «anticonformistes » pour reprendre un terme utilisé par René Nelli. Il est donc possible d'intégrer la scatologie dans une réflexion sur le discours amoureux des troubadours. On peut postuler que la scatologie a pour point commun essentiel avec l'obscénité la mise en scène discursive d'une réalité refoulée par un processus de civilisation ${ }^{3}$. Un parallèle peut être fait avec les passions mélancoliques que l'on cherche à contrôler, à réprimer ou à sublimer ${ }^{4}$. On considérera également que la

\footnotetext{
${ }^{1}$ Écrivains anticonformistes du Moyen Âge occitan, 1, La Femme et l'amour, René NELLi (éd.), Paris, Phébus, 1977.

${ }^{2}$ Burlesque et obscénité chez les troubadours : le contre-texte au Moyen Âge, Pierre BEC (éd.), Paris, Stock, « Moyen Âge », 1984.

${ }^{3}$ Cf. Norbert Elias, La Civilisation des moeurs, Paris, Calmann-Lévy, 1973.

${ }^{4} \mathrm{Cf}$. Pierre LeVron, Naissance de la mélancolie dans la littérature des XII et XIII ${ }^{e}$ siècles, thèse de doctorat (inédite) dirigée par Jacqueline Cerquiglini-Toulet et soutenue devant l'Université de Paris-Sorbonne le 30 juin 2005.
} 
scatologie est un objet narratif dynamique ${ }^{5}$, parce qu'elle est le moteur d'un discours poétique fondé sur l'inversion des codes de bienséance. Elle est en outre un objet de recherche méritant des investigations plus approfondies : le Moyen Âge est peu présent dans Pipi, Caca, Popo de Jean Feixas, qui propose une mise en perspective historique générale de la scatologie $^{6}$. Il paraît possible d'émettre l'hypothèse que ces textes ne relèvent pas seulement de la parodie ou d'un certain défoulement poétique, mais qu'ils s'insèrent dans les discours sur l'amour courtois. Il semble en effet que la différence entre ces textes scatologiques et les poèmes «réguliers » réside principalement dans la stratégie rhétorique de leurs auteurs. Ils peuvent donc nous aider à cerner avec plus de précision l'essence de la mélancolie amoureuse si caractéristique de cette érotique. Le discours scatologique concerne trois domaines: les normes sociales (dont les canons de la beauté), le service d'amour et les connaissances médicales.

\section{Courtoisie et scatologie, ou de la beauté de la scatologie}

Le discours amoureux courtois a deux directions essentielles: l'expression du désir, mais aussi l'érection de l'aimée en référence, parce qu'elle incarne les qualités que l'on attend d'une dame. C'est ce qui explique l'importance du complexe unissant la beauté de la dame à ses vertus dans les textes orthodoxes. Qu'en est-il dans la lyrique de la scatologie? On y rencontre des portraits repoussants de la dame. La Porquiera, gardeuse de porcs, décrite dans un poème anonyme des Leys $d^{\prime} A m o r^{7}$ est $«$ negre cum pega ${ }^{8}$ et son langage n'est pas plus raffiné que

\footnotetext{
${ }^{5}$ Voir dans ce bulletin la contribution de Francesco MONTORSI « Quelques pistes de réflexions pour une étude scatologique », p. 35-54.

${ }^{6}$ Jean FEIXAS, Pipi, caca, popo : histoire anecdotique de la scatologie, Genève, Liber, 1996, p. 50-56.

${ }^{7}$ La Porquiera, in Burlesque et obscénité chez les troubadours, éd. cit., p. 184-190.
} 
son apparence :

En est mieg, jos sa gonèla,

Se grata fòrtment e brega

Lo sieu corsegàs mal fach :

Si'l pans non fos del gannach,

Peregra'l tota la plega!

E respondèt entertan,

Amb boaral votz grossiera :

«Om, perqué'm vas enujan?

Sèc, de per Dieu, ta carriera ! ${ }^{9}$

Si le motif de l'aimée inflexible éconduisant l'amoureux est parodié ici, la scatologie vise le comportement du personnage. La dame représentée en majesté dans le discours courtois traditionnel est remplacée par une figure parlant sur un ton grossier et grattant son intimité. Trois éléments apparaissent: la laideur, la mauvaise éducation et l'absence d'hygiène. Par-delà l'amusement du public, deux enjeux se dessinent pour ce poème : sa versification, qui motive son insertion dans le traité, puisqu'il s'agit de fournir un exemple de composition poétique ${ }^{10}$, et la domination symbolique du troubadour. L'accomplissement formel de la canso fait de celui qui la compose le maître du beau langage. L'homme de lettres à la technique prosodique affirmée montre sa supériorité sur la paysanne en faisant d'elle simultanément un être subalterne, que l'on peut séduire comme dans une pastourelle, et une figure grossière, dépourvue d'une belle rhétorique et de belles manières. La scatologie devient un instrument de mise à distance : elle oppose le troubadour au langage noble à une femme du peuple dont l'infériorité sociale est définie par sa laideur et par la

\footnotetext{
8 « Noire comme de la poix », ibid., v. 8.

9 «Entre-temps, elle gratte et frotte avec vigueur son gros corps mal fait sous sa gonnelle. S'il n'y avait pas eu le pan de la garnache, on aurait pu voir toute sa fente ! Elle répondit en même temps d'une grosse voix de bouvier: "Homme, pourquoi es-tu en train de m'ennuyer? Au nom de Dieu, passe ton chemin !" », ibid., v. 18-26.

${ }^{10}$ Le poème sert d'exemple aux coblas retrogradas per acordansa, dans lesquelles la rime orpheline du dernier vers d'une cobla est reprise dans la suivante ; la versification est ensuite inversée. On a le schéma suivant dans ce poème : ababc-cdd-cee-ffg puis g-ffee-cdd-baba.
} 
rusticité de son comportement. Il n'est pas non plus impossible d'y voir l'autodérision d'un troubadour méprisé par une ignorante aussi caricaturale.

Il arrive également que la scatologie souligne un décalage plus complexe entre la norme et ce que l'on chante. Guillem de Berguedan l'emploie dans un sirventès satirique visant un clerc fornicateur :

La mesquina

Flaira e grina,

Que maistre Rogier enclina,

Tan festina

E s'aisina

Tro sent la douçor conina ;

De s'orina

Fai mezina,

E'l con non cessa ni fina,

Qu'en la cuitat peitavina

Se rompèt fotent l'esquina. ${ }^{11}$

Le poème utilise les registres de l'obscénité et de la scatologie. Liées par l'ondinisme, elles construisent un paradigme du scandale touchant aussi bien la jeune fille insatiable que le clerc obsédé par le fag, c'est-à-dire par l'amour physique, couronnement désiré de la fin'amor. L'élément scatologique ridiculise tout d'abord le personnage ; il le place ensuite dans une posture opposée à sa fonction. L'inversion courtoise et sensuelle déjà présente dans la canso de Raimbaut d'Orange Ar resplan la flors enversa... ${ }^{12}$ cède la place à une sorte d'écrasement dans lequel l'amant est

11 «La jeune fille que maître Roger tient courbée sous lui halète et gémit. Elle s'affaire tant et elle se met tant à l'aise qu'elle finit par sentir la douceur du con. Elle fait de son urine un remède, et son con n'a ni trêve ni repos : elle s'est brisée le dos en baisant à Poitiers. », GUILLEM DE BERGUEDAN, Un trichaire..., in Burlesque et obscénité chez les troubadours, éd. cit., p. 133-137, v. 23-34.

12 Raimbaut D'ORAnge, Ar Resplan la flors enversa..., in Anthologie des Troubadours, Pierre BEC (éd.), Paris, UGE, 1979, «10/18», p. 148-154. Cette canso exploite le thème de l'inversion (comme le montre l'emploi de l'adjectif enversa, « inversée » «à l'envers », du verbe (en)versar, « renverser») - métaphore de la révolution des qualités intrinsèques propre à la mélancolie amoureuse - en l'appliquant successivement à la saison de la floraison, l'hiver (I, v. 2-7), à la sensibilité de l'amant puis à celle de l'être fou (II, v. 9, et V, v. 33), aux lausengiers, adversaires de l'amant et de la dame (III, v. 1-7) et à la dame, que l'amant enversa en l'embrassant (IV, v. 25). 
avili par une pratique sexuelle scandaleuse. Il s'agit alors de dénoncer la saleté morale de frère Roger en le montrant en train de commettre un acte ignoble.

Ces variations sur une scatologie discriminante, mettant en scène des figures scandaleuses ou illégitimes opposées à un locuteur incarnant plus ou moins implicitement les normes communes, ne doivent pas masquer une autre fonction du discours scatologique: la description de la souffrance amoureuse de l'amant. Une cobla parodiant la canso de Bernart de Ventadorn Quan la freid' aura venta... le montre :

Quan lo petz del cul venta

Dont Midònz caga e vis,

Vejaire m'es qu'eu senta

Una pudor de pis

D'una orrida sangnenta

Que tot jorn m'escarnís,

Qu'es mais de petz manenta

Que de marabodís,

E quan jatz [sus] son pis,

Plus put d'autra serpenta. ${ }^{13}$

Le comique repose sur l'inversion du souffle : le motif de l'air paradisiaque venant de l'endroit où vit l'aimée est transformé en celui du pet qu'elle émet elle-même. La dame est d'ailleurs réduite à une série d'émissions scatologiques faites de vents et d'urine. La tension entre ces fluides extrêmement malodorants et méphitiques et la courtoisie du terme «Midònz » montre tout d'abord que la dame conserve une posture autoritaire : elle émet des signes que l'amant reçoit et interprète, comme dans une canso conventionnelle. Ce conflit entre des émissions scatologiques et un terme courtois oppose ensuite l'amour à des

\footnotetext{
13 «Quand le pet du cul vente de l'endroit où ma dame chie et pisse, j'ai l'impression de sentir la puanteur de la pisse d'une affreuse saignante qui ne cesse de se moquer de moi et qui est plus riche de pets que de maravédis. Et quand elle est couchée sur sa pisse, elle pue plus que n'importe quelle autre serpente. ", Quan la freid' aura venta..., in Burlesque et obscénité chez les troubadours, éd. cit., p. 173-175, v. 1-10.
} 
sollicitations sensorielles pénibles ; la mélancolie amoureuse est révélée par une puanteur qui se substitue littéralement à des paroles méprisantes et orgueilleuses. Le discours scatologique exprime une souffrance atrabilaire provoquée par un traumatisme psychique qu'il métaphorise en rendant très explicite la cause de la souffrance. Le pet est une image du verbe orgueilleux. Il arrive que le rot assume cette fonction, comme dans la sotte chanson Chans de singe ne poire mal pellee... ${ }^{14}$. Le discours scatologique ne se limite toutefois pas à cela car il participe d'une réflexion sur la nature et la légitimité du service d'amour.

\section{Le service d'amour scatologique}

Le motif $\mathrm{du}$ «pet faisant marcher les navires ${ }^{15}$ est exploité par un partimen opposant Arnaut Catalan au comte de Provence ${ }^{16}$. Ce dernier demande au troubadour s'il serait prêt à émettre une flatulence assez puissante pour propulser un bateau emprunté par des dames de la noblesse se rendant outre-mer ${ }^{17}$. Le troubadour répond par l'affirmative :

«Sénher En Coms, eu ai un tal usatge

Qu'adès mantenh dòmnas e drudaria ;

E si be'l petz no'm vengués d'agradatge,

Faria lo, car si eu no'l fazia,

Falhiria vas dòmnas malamen.

E dic vos ben que s'ilh per altramen

Non podion anar a salvamen

Après lo pet, totz me concagaria. ${ }^{18}$

${ }^{14}$ Chans de singe ne poire mal pellee..., in Chansons des Trouvères : Chanter m'estuet, Samuel N. Rosenberg et Hans Tischler (éd.), Paris, LGF, « Le Livre de Poche. Lettres gothiques », 1995, p. 254-257.

${ }^{15}$ C'est le titre donné par Pierre BEC au paragraphe regroupant les deux partimens sur ce thème. Cf. Burlesque et obscénité chez les troubadours, éd. cit., p. 154.

16 Amics N'Arnautz..., in Burlesque et obscénité chez les troubadours, éd. cit., p. 154-156.

${ }_{17}$ Ibid., première cobla, v. 1-8.

18 «Seigneur comte, j'ai une habitude bien ancrée : je défends toujours les dames et la courtoisie. Et même si le pet ne me venait pas de mon plein gré, je le ferais, car je commettrais une grave faute envers les dames en m'en abstenant. Et je vous dis bien 
Le débat oppose la mesure défendue par le comte à l'humiliation de l'homme courtois défendue par Arnaut. La scatologie revêt une valeur casuistique et une valeur rhétorique. Dans le premier cas, elle relève des aboutissements extrêmes du service amoureux, qui comprennent souvent l'humiliation de l'amant. Il est possible de comparer ce pet propulseur à d'autres épreuves du service des dames. Comme Lancelot montant sur la charrette chez Chrétien, le servant des dames abdique sa dignité d'homme noble. La radicalité de cette épreuve est déjouée par deux éléments : la valeur hypothétique du cas, soulignée par le comte, et la nature même de l'épreuve, dont le caractère surnaturel renvoie à certains gabs faisant intervenir un souffle prodigieux. Le Pèlerinage de Charlemagne montre ainsi Roland prétendant faire se heurter les portes de Jérusalem et faire tomber les parures et la barbe du roi Hughes en soufflant dans une trompe $^{19}$. Les deux textes présentent d'ailleurs un important parallélisme formel, puisqu'ils se placent dans le cadre d'une plaisanterie autorisée par un personnage de référence. Le pet relève ainsi d'un discours humoristique et ludique. Les réactions du comte, qui dénoncent le scandale et la grossièreté des propos du troubadour ${ }^{20}$, soulignent toutefois une tension entre l'amusement licite et la subversion des normes morales et langagières : réprimé par une posture autoritaire, le pet s'apparente à une sorte de retour du refoulé alors que l'éthique courtoise prône les belles manières auxquelles le service des dames s'oppose ici.

Un second partimen opposant sur ce thème Arnaut au roi Alphonse $\mathrm{X}$

que s'il n'y avait pas d'autre manière pour les faire atteindre leur salut, je me conchierais après le pet. », ibid., seconde cobla, v. 9-16.

${ }^{19}$ Le Voyage de Charlemagne à Jérusalem et à Constantinople, Paul AEBISCHER (éd.), Genève, Droz, 1965, v. 469-483.

20 «Amics N'Arnautz, tròp parlatz malamen / Et auretz ne gran blasme de la gen, / (Que) passar voletz tan bèl còrs avinen / Ab vent de cul en tèrra de Suria » («Seigneur et ami Arnaut, vous parlez très grossièrement et vous en recevrez grand blâme des gens ! Vous voulez faire passer par un vent de cul tant de belles et gracieuses créatures en terre de Syrie!»), Amics N'Arnautz..., in Burlesque et obscénité chez les troubadours, éd. cit., troisième cobla, v. 17-20. 
de Castille remet lui aussi en question la courtoisie réelle de ce service :

«Dom Arnaldo, fostes errar

Por passardes com batares

Vossa senhor a ultramar,

Que non cuyd' eu que i a tres

No mundo de tam gran valor,

E juro vos par sam Vincente

Que non é boon doneador

Quen esto ferez a çyente. » ${ }^{21}$

Le scandale ne réside pas tant dans le caractère choquant du pet que dans la disproportion entre la valeur morale des dames et le service qu'on leur rend. L'enjeu n'est pas l'humiliation de l'amant, mais sa capacité à servir avec discernement. La notion d'un miracle plaisant dont une personne élue mérite de bénéficier est beaucoup plus nette que dans le poème précédent, puisqu'elle souligne l'erreur que constitue dans l'orthodoxie courtoise un service dont toutes les dames bénéficieraient. Moins axée que la pièce du débat avec le comte de Provence sur la folie amoureuse d'un amant courtois abdiquant sa dignité, la tenso met en évidence le souci d'un service intelligent et proportionné aux vertus de la dame servie. Elle interroge plus indirectement la légitimité d'une lyrique que toutes et tous peuvent entendre, quels que soient leurs mérites en amour. Argument saisissant et amusant, le pet pourrait être un avatar éloigné du trobar leu, parce que tout le monde s'en amuse, tout comme les cansos composées « d'une manière facile» (ou trobar leu) peuvent être reprises par tout le monde, même par les femmes allant chercher de l'eau à la fontaine, comme l'affirment certains troubadours. L'interlocuteur, tout aussi frotté de poésie que bénéficiant d'une posture autoritaire, pourrait alors défendre l'optique propre au trobar ric d'un chant compréhensible par les seuls interlocuteurs

21 «Seigneur Arnaud, vous déraisonnez à vouloir faire passer votre dame outre-mer grâce à des pets. En effet, je ne pense pas qu'il y en ait trois au monde de si grande valeur ! Et je vous jure par saint Vincent qu'il n'est pas un bon amant, celui qui fait cela volontairement. ", Sénher, adars ie'us venh querer..., in Burlesque et obscénité chez les troubadours, éd. cit., p. 157-160, quatrième cobla, v. 25-32. 
savants et instruits.

$$
\text { L'«affaire Cornilh » }{ }^{22} \text { présente une autre question de casuistique }
$$
amoureuse, reposant sur la distance entre l'épreuve et le fait. Une dame a demandé à son amant Bernart de Cornilh de lui souffler dans le derrière ; il s'y est refusé. Quatre sirventès, composés par Arnaut Daniel ${ }^{23}$, Truc Malec $^{24}$, Raimon de Durfort ${ }^{25}$ et Bernart de Durfort ${ }^{26}$ forment une polémique plaisante. Arnaut Daniel prend la défense de l'amant :

« Ben l'agr'obs que fos becutz

E-1 becs fos loncs et agutz,

Que-1 corns es fers, laitz e pelutz

E nul jorn no estai essutz,

Et es prions dins la palutz,

Per que rellent' en sus lo glutz

C'ades per si cor ne rendutz;

E non voill que mais sia drutz

Cel que sa boch al corn condutz. ${ }^{27}$

Raimon de Durfort conteste la saleté de la situation :

«Cornatz lo corn, qu'ayssi lo-us vir,

Qu'ieu l'ai fach lavar e forbir,

E ja no-l sentiretz pudir,

Et ja no tematz escarnir. ${ }^{28}$

Les troubadours utilisent une épreuve scatologique relativement répandue

${ }^{22}$ D’après les titres donnés par Pierre BEC (« Peut-on jouer du cor dans le derrière des dames ? L'affaire Cornilh», in Burlesque et obscénité chez les troubadours, éd. cit., p. 138) et René NELLI («Autour de l'affaire Cornilh », in Écrivains anticonformistes..., éd. cit., p. 86) aux paragraphes regroupant les poèmes composés sur cette anecdote par Arnaut Daniel, Raimon et Bernart de Durfort et TruC Malec.

${ }^{23}$ ARnaUt Daniel, Pois Raimons e'n Truc Malecs..., in Écrivains anticonformistes $d u$ Moyen Âge occitan, éd. cit., p. 82-85.

${ }^{24}$ TRUC MALEC, En Raimon, be-us tenc a grat..., ibid., p. 86.

${ }^{25}$ RAIMON DE DURFORT, Truc Malec, a vos me tenh..., ibid., p. 86-90.

${ }^{26}$ BERNART DE DURFORT, Ben es malastrucx dolens..., ibid., p. 90-95.

${ }^{27}$ «Il aurait bien besoin d'avoir un bec, et que ce dernier fût long et aigu, car le cor est rude, laid et poilu et n'est jamais sec. Et c'est profond dans ce marécage, parce que la poix qui sans cesse s'en échappe d'elle-même y fermente. Et je ne veux pas qu'il soit aimé un jour, celui qui colle sa bouche à un tuyau pareil! », ARNAUD DANIEL, Pois Raimons e'n Truc Malecs..., ibid., seconde cobla, v. 11-19.

${ }_{28}$ «Soufflez dans le cor! Voyez: je le tourne vers vous! Je l'ai fait laver et astiquer! Vous ne sentirez aucune puanteur et ne craignez pas de tromperie de ma part!», RAIMON DE DURFORT, Truc Malec, a vos me tenh..., ibid., troisième cobla, v. 21-24. 
dans la littérature comique médiévale : l'exhibition du postérieur. Ils en retiennent deux aspects. L'emprise, tout d'abord, de la dame sur son amant, qui rappelle l'humiliation amoureuse évoquée plus haut. René Nelli attire l'attention sur le caractère ambivalent d'une épreuve susceptible de révéler le pouvoir de l'aimée sur l'amant ou de le repousser ${ }^{29}$. On se rapproche de l'usage de la «monstrance du cul » comme ruse, attesté par le Roman de Renart $^{30}$, dans la mesure où la dame peut avoir trouvé cet expédient pour éconduire le malheureux Bernart! Les auteurs utilisent également cette exhibition comme un révélateur de la nature véritable d'un personnage que sa lâcheté transforme en victime ${ }^{31}$. Lâcheté qu'Arnaut Daniel conteste alors qu'elle est soulignée par son adversaire. La spécificité de la controverse réside dans le débat suscité par l'événement. Arnaut Daniel met l'accent sur un postérieur révélant un corps féminin à l'intérieur répugnant, témoin de l'immoralité de l'aimée, et en vient à évoquer une véritable laideur mélancolique intérieure qui s'oppose à la séduction. Atrabilaire, l'intérieur du cor l'est par son aspect effrayant. Le troubadour décrit un milieu hostile, caractérisé par des processus chimiques produisant une substance grossière et par sa capacité à retenir prisonnière la victime qui s'y enfonce. Raimon de Durfort décrit quant à lui l'extérieur, dont la propreté s'assimile à la beauté de la dame. La communion courtoise annonce chez lui l'acte sexuel dont elle est une version atténuée. Arnaut Daniel, au contraire, décrit la démesure d'une dame qui aurait pu préférer une épreuve moins grossière et

\footnotetext{
${ }^{29}$ Écrivains anticonformistes du Moyen Âge occitan, éd. cit., p. 80.

${ }^{30}$ Le Roman de Renart, Armand Strubel (éd.), Paris, Gallimard, « Bibliothèque de la Pléiade », 1998, Branche XXII, La monstrance du cul, p. 745-749. La monstrance du cul est une épreuve destinée à attribuer un jambon qu'un paysan, Ysengrin et l'ours Patous veulent partager. L'épouse du paysan obtient la charcuterie en se faisant passer pour son mari et en montrant son anus et son vagin de manière à ne laisser voir qu'un trou unique. L'ours et le loup s'enfuient !

${ }^{31} \mathrm{Cf}$. Berengier au lonc cul, in Chevalerie et grivoiserie : fabliaux de chevalerie, Jean-Luc LeClanche (éd.), Paris, Champion, 2003, p. 84-101.
} 
plus subtile ${ }^{32}$. Si l'exacerbation de la cruauté de la dame rend le refus légitime aux yeux d'Arnaut, elle est niée par Raimon, qui met l'accent sur la dimension ludique de l'épisode. Les deux troubadours s'opposent sur la nature d'une épreuve dont le caractère scatologique est très ambivalent. Le débat tourne donc autant autour d'une définition subjective de ce qui est scatologique que de la valeur de l'épreuve, la première problématique donnant une réponse morale à la seconde: l'acte est d'autant plus disqualifié qu'on peut le juger scatologique. La grossièreté n'est pas seule en cause et peut-être l'hypothèse de l'influence de conceptions médicales mérite-t-elle dès lors d'être examinée.

\section{Scatologie et discours médical}

Pourquoi terminer notre enquête en parlant d'un probable substrat médical ? Ces poèmes s'insèrent tous dans un discours normatif sur la femme et l'amour. Loin de subvertir les conceptions dominantes, ils les appuient. Nous venons de souligner deux aspects essentiels d'une vision scatologique de la femme, d'une part sa saleté et d'autre part son aptitude à devenir une figure inquiétante. Il faut étudier maintenant la relation que les éléments du discours scatologique entretiennent avec le savoir médical. On peut aussi s'interroger sur l'usage ambivalent d'une scatologie qui amuse les destinataires de ces pièces mais qui participe également d'une problématique autrement plus sérieuse, celle de la guérison d'une mélancolie d'amour maladive. La parodie de Quan la freij'aura venta de Bernart de Ventadorn évoque, tout comme le sirventès d'Arnaut Daniel sur l' " affaire Cornilh », le sang menstruel. Un milieu très humide est décrit dans la cobla parodique. Trotula affirme dans son De Mulieribus

\footnotetext{
32 «Pro hi agra d'autres assais / de plus bels e que valgron mais.» (« il y aurait beaucoup d'autres épreuves / bien plus belles et qui vaudraient davantage. »), ARNAUT DANIEL, Pois Raimons e'n Truc Malecs..., in Écrivains anticonformistes du Moyen Âge occitan, éd. cit., troisième cobla, v. 12-13.
} 
passionibus ante in et post partum que les menstrues purgent la femme d'une humidité excessive ${ }^{33}$. L'alliance du sang à l'urine présente dans la parodie de Bernart de Ventadorn existe également chez Arnaut Daniel :

Mieills li fora fos en issill

Qu'el la cornes en l'efonill

Entre l'eschin'e-l penchenill,

Per on se segon li rovill ;

Ja non saubra tant de gandill

No-il compisses lo groing e-1 cill. ${ }^{34}$

La «rouille » évoquée par le poète s'intègre à un système médiéval de pensée étymologique et symbolique ${ }^{35}$. Le sang menstruel évoqué par nos deux troubadours s'éloigne cependant de la fonction purificatrice pour devenir un précipité inquiétant et menaçant.

Réduit à l'anus, le corps féminin est simultanément sale et terrifiant. La conjonction avec la mélancolie s'opère sur ce point. On retrouve en effet partiellement une définition de la mélancolie, ou bile noire, proposée par le Breviari d'Amor de Matfre Ermengaud :

[...] pueis fai la melencolia de sso quez es sec ab frejor terrenal, ab mens de valor $;^{36}$

L'élément fondamental de l'exposé est cette idée de bile noire constituée d'un précipité de nutriments peu utiles. L'encyclopédiste ne parle pas de saleté, nous objectera-t-on. L'idée d'un fluide impur n'en existe pas moins dans sa démonstration qui associe la valeur symbolique et morale de

${ }^{33}$ Cf. Danielle JACQUART et Claude ThOMASSET, Sexualité et savoir médical au Moyen Âge, Paris, PUF, « Les Chemins de l'histoire », 8, 1985, p. 98.

34 «Il lui aurait mieux valu de partir en exil que de souffler dans l'entonnoir de la dame, entre le dos et le pénil, là où s'échappe la "rouille". Il n'aurait jamais pu tant se protéger qu'elle ne lui pisse pas sur le groin et le sourcil. », ARNAUT DANIEL, Pois Raimons e'n Truc Malecs..., in Écrivains anticonformistes du Moyen Âge occitan, éd. cit., cinquième cobla, v. 40-46.

${ }^{35}$ Cf. Danielle JACQUART et Claude ThOMASSET, Sexualité et savoir médical au Moyen Âge, op. cit., p. 102.

36 «Ensuite, la bile noire se sert de ce qui est froid, sec, terrestre et qui a le moins de valeur [nutritive]. », Le Breviari d'Amor de Matfre Ermengaud, Peter T. RICKETTS (éd.), t. II, 1-8880, London, Westfield College, «A.E.I.O. », 1989, v. 7746-7748. 
l'humeur à l'utilité et à la propreté de ses composants. Très éclairante est l'opposition implicite entre le flegme et la bile noire présente dans les vers 7735 à 7748. Les deux humeurs constituent les extrémités d'un développement qui décrit la manière dont les aliments sont digérés et répartis entre les quatre fluides humoraux : le sang, le flegme, la bile jaune et la bile noire. Le flegme est une humeur froide, «haute», nourrie d'aliments peu digérés, mais l'auteur n'exprime pas à son sujet de jugement dépréciatif ${ }^{37}$; la bile noire, à l'inverse, appelle ce dernier. Humeur néfaste, elle s'oppose alors à trois humeurs neutres ou positives. Le discours les discrimine par le contraste entre la neutralité du flegme et la grossièreté implicite de la bile noire, qui est lourde parce qu'elle est traditionnellement associée à la terre. Beaucoup plus radical que le clivage que Jean-Marie Fritz a mis en évidence entre ces deux humeurs ${ }^{38}$, le discours savant structure une démonstration médicale et morale où la mélancolie est isolée par sa saleté du reste du système humoral. L'intimité anale est donc le lieu par où s'échappent des liquides qui servent de miroirs à cette humeur qui leur ressemble. Arnaut Daniel ajoute un autre élément par l'évocation de la déviation sexuelle, représentée par un ondinisme menaçant. Le parallélisme entre la bile noire et les déjections s'opère également par l'assimilation symbolique du noir du teint atrabilaire et de la couleur foncée des excréments. Jean Starobinski avait formulé l'hypothèse que les médecins de l'Antiquité avaient déduit l'existence de la bile noire en observant des selles de cette couleur ${ }^{39}$. Le Breviari d'Amor de Matfre

\footnotetext{
37 «Quar natura premieiramen / d'aquo quez es freg et humit / e mens cueg e mens digirit / la flegma tantost congria » («Car la nature commence par apporter ce qu'il lui faut au flegme : ce qui est froid et humide et moins cuit et moins digéré »), ibid., v. 7736-7739.

${ }^{38}$ Jean-Marie FRITZ, « La théorie humorale comme moyen de penser le monde : limites et contradictions du système », in Dominique BOUTET et Laurence HARF-LANCNER (dir.), Écritures et modes de pensée au Moyen Âge, VIII ${ }^{e}-X V^{e}$ siècles, Paris, Presses de l'École normale supérieure, 1993, p. 13-26. Cf. le schéma p. 19.

${ }^{39}$ Jean STAROBINSKI, Histoire du traitement de la mélancolie des origines à 1900, Bâle,
} 
Ermengaud décrit ainsi l'atrabilaire :

Melencolicz per natura

es de mout laja figura,

quar deu haver color de brac

e portar pro de mal el sac. ${ }^{40}$

On retrouve ici deux éléments importants du discours scatologique des poèmes d'oc: le teint, littéralement boueux, dont la coloration brunâtre évoque éventuellement celle des déjections, et la laideur. Le discours scatologique et le discours sur la mélancolie décrivent tous les deux un phénomène choquant.

Si la canso révèle l'amour secret pour une dame au corps parfait, le chant scatologique parodiant les codes du lyrisme amoureux exhibe la chimie interne grossière de la femme. Le corps sale qui émet vents, urines, sang menstruel et excréments est symétrique au «corps courtois » idéalisé et érotisé. Les parodies de prototypes conventionnels permettent même d'avancer l'hypothèse que ce corps scatologique en serait un avatar. Là encore, les théories médicales du Moyen Âge central peuvent contribuer à l'explication de ces poèmes. Le «contre-corps » scatologique peut être laid, comme on le voit avec la canso de la Porquiera :

Et ac son còrs fèr e lag,

Escur e negre cum pega ;

Gròssa fo coma tonèla,

Et ac cascuna mamèla

Tan gran que semblèt Englesa.

Ieu, que la vi malsabesa,

Cazèt mi tota la braça. ${ }^{41}$

Laboratoire Geigy, «Acta Psychosomatica », 3, 1960.

40 «Le mélancolique par nature est de très laide figure, parce qu'il doit avoir un teint de boue et porter beaucoup de mal dans sa besace. », Le Breviari d'Amor de Matfre Ermengaud, éd. cit., v. 7779-7782.

41 «Et elle était farouche et laide, sombre et noire comme de la poix, grosse comme un tonneau. Et ses deux seins étaient si gros qu'elle ressemblait à une anglaise. Quant à moi, dès que je la vis si dégoûtante, mes deux bras m'en tombèrent », La Porquiera, in Burlesque et obscénité chez les troubadours, éd. cit., p. 184-190, première cobla, v. 7-13. 
La laideur a partie liée avec la scatologie, puisqu'elle renvoie à la complexion mélancolique du personnage, renforcée par son appartenance à la paysannerie et par son association avec les porcs, dont la viande favorise la bile noire selon les médecins médiévaux ${ }^{42}$. Moins scatologique à proprement parler est la réaction émotionnelle du troubadour. Elle n'en est pas moins révélatrice d'un choc provoqué par la déception de l'horizon d'attente poétique: la laide porchère est le miroir inverse de la belle bergère. Le lien avec la scatologie se fait justement à cet endroit où le discours révèle ce qui est initialement caché, la porchère étant tout d'abord à l'abri des regards. Loin de vouloir faire simplement rire, ces corps hideux peuvent relever d'une volonté clinique, celle de guérir l'illusion amoureuse. Le Lilium Medicinae de Bernard de Gordon conseille de soigner de la sorte un amour héroïque pour lequel toutes les cures alternatives auront échoué :

Finaliter autem cum aliud consilium non habemus: imploremus auxilium et consilium vetularum, ut ipsam dehonestent et difament quantum possunt. Ipsae enim habent artem sagacem ad hoc plus quam viri. Cum dicit Auicenna aliqui sunt qui gaudent in audiendo fetida et illicita. Quaeratur igitur vetula turpissima in aspectu cum magnis dentibus et barba : et cum turpi et vili habitu : et quod portet subtus gremium pannum menstruatum et aduienens philocapta quod incipiat dehonestare camisiam suam dicendo : quomodo est tignosa et ebriosa : et quod mingit in lecto : et quod est empileptica et impudica : et quod in corpore suo sunt excrescentiae enormes cum fetore anhelitus, et aliis omnibus enormibus in quibus vetulae sunt edoctae. Si autem ex his persuasionibus nolit dimettere: subito extrahat pannum menstruatum coram facie : portando dicendo clamendo : talis est amica tua talis. Et si ex his non dimiserit : iam non est homo sed diabolus incarnatus. ${ }^{43}$

\footnotetext{
${ }^{42}$ Cf. Aldebrandin De Sienne, Le Régime du corps, Roger LANDOUZY et Louis PePIN (éd.), Paris, Champion, 1911, p. 122.

43 «Pour finir, quand nous n'avons pas d'autre solution, implorons l'assistance et le conseil des vieilles femmes, afin qu'elles diffament et déshonorent l'aimée autant qu'elles le peuvent. Bien plus que les hommes, elles ont une compétence redoutable en la matière. Comme le dit Avicenne : "il y en a certaines qui se réjouissent d'entendre des propos illégitimes et dégoûtants". Que l'on aille donc chercher une vieille à l'aspect
} 
Les soins se fondent sur la mise en évidence des éléments horrifiants décrits par les poèmes scatologiques. Nous retrouvons la laideur d'un substitut de l'aimée, la vulgarité de son discours, le sang menstruel et l'intimité féminine sale. Comme dans la lyrique, l'horizon d'attente de l'amant est déçu. Comme dans les cansos et sirventès scatologiques, le spectacle de la laideur et de la saleté est une construction et le thérapeute se rapproche fonctionnellement du troubadour. Il s'en éloigne cependant par un élément fondamental car la mise en scène est visible tout autant qu'audible. Il ne concrétise pas seulement une dynamique, présente par exemple dans l'Altrier cuidèi aver druda $a^{44}$ où l'amant perd l'envie d'aimer, mais construit un spectacle extrêmement concret qui inverse l'emballement intellectuel et passionnel propre à la mélancolie amoureuse, là où les troubadours proposent des «contre-modèles» destinés à déjouer les déviations de l'esprit ${ }^{45}$. Le registre scatologique reste donc intellectualisé chez les poètes et nous n'avons d'ailleurs pas rencontré de cas médicaux ou littéraires soignés par le chant de poèmes de ce genre, alors que, selon Bernard de Gordon ${ }^{46}$, la sensibilité des amants héroïques à la poésie est très

affreux avec de la barbe et de grandes dents ainsi que des vêtements laids et vulgaires. Qu'elle porte, en outre, un linge menstruel dans son giron. Qu'elle vienne devant l'amant en se mettant à malmener ses habits et disant : "Celle-là, c'est une teigneuse et une ivrognesse ! Et elle pisse au lit ! Et elle est impudique et épileptique ! Et il y a dans son corps d'énormes tumeurs pleines de pestilence!" Et toutes ces énormités dans lesquelles les vieilles femmes sont très expertes. Si cela ne parvenait pas à affaiblir ses convictions, qu'elle sorte le linge menstruel et le mette devant le visage de l'amant; qu'elle s'exclame en le tenant: "Elle est comme ça, ton amie, elle est comme ça !" Et s'il ne renonce pas à son amour après tout cela, ce n'est plus un homme du tout, mais un diable incarné. », BERNARD DE GORDON, Lilium Medicinae, in John Livigston LOWES, «The Loveres Maladye of Hereos », Modern Philology, 11 (1914), p. 501.

${ }^{44}$ Il s'agit d'un poème parodique (mais non scatologique) du XIII ${ }^{\mathrm{e}}$ siècle dans lequel un amant prend la fuite après avoir rencontré son amie «ben parlant d'amor» mais... d'une laideur repoussante! Cf. Burlesque et obscénité chez les troubadours, éd. cit., p. $179-183$.

${ }^{45}$ Le médecin et les troubadours jouent sur l'imagination. Cf. Giorgio AGAMBEN, Stanze : parole et fantasme dans la culture occidentale, Paris, Payot, « Rivages », 1994, p. 189.

46 «Et si audiant cantilenas de separatione amoris statim incipiunt flere et tristari. Et si 
vive et qu'il est bon de jouer de la musique devant eux ${ }^{47}$. Il semble que les usages médicaux et poétiques du temps ne le permettent pas : lourdement investie de propriétés mélancoliques parce qu'elle renvoie aussi bien à l'aliénation amoureuse qu'à une corporalité angoissante, la scatologie présente le risque de saturer l'esprit de l'amant par des passions atrabilaires contradictoires. Son emploi lyrique sera donc préventif : le public de ces textes est mis en garde contre les illusions amoureuses par une matérialité aussi agressive qu'envahissante.

La scatologie des troubadours a pour fonction essentielle de prévenir et de combattre une illusion amoureuse génératrice de mélancolie. Reflet poétique d'une bile noire qu'il faut tenir à distance, elle repose sur une tension entre la peur qui suinte et le rire qui doit la déjouer. Le chant scatologique diffère donc fondamentalement du chant courtois par ce conflit qui se substitue à celui qui oppose désir et crainte de l'aimée. La parole libère de l'angoisse, elle n'en délivre pas totalement. Il n'y a donc pas d'hétérodoxie véritable dans ces poèmes dont le ton tranche avec celui de la grande lyrique courtoise. La proximité dynamique de ces textes avec des pièces beaucoup plus classiques est un principe essentiel définissant le « contre-texte » qui reposerait alors sur une mise en scène de tensions entre les aspirations et les craintes d'une manière beaucoup plus violente que dans la lyrique courtoise.

audiant de coniuctione amoris statim incipiunt ridere et cantare. » («Et s'ils écoutent des chansons parlant d'amants qui se séparent, ils se mettent aussitôt à pleurer et à s'attrister; s'ils en écoutent qui parlent d'amants qui s'unissent, ils se mettent sur-lechamp à rire et à chanter. »), BERNARD DE GORDON, Lilium Medicinae, éd. cit, p. 500.

${ }^{47}$ Ibid., p. 501. 